\title{
Symmetry energy from the nuclear collective motion: constraints from dipole, quadrupole, monopole and spin-dipole resonances
}

\author{
G. Colò ${ }^{1,2}$, U. Garg ${ }^{3,4}$, H. Sagawa ${ }^{5,6}$ \\ 1 Dipartimento di Fisica, Università degli Studi di Milano, via Celoria 16, I-20133 Milano, Italy \\ 2 INFN, sezione di Milano, via Celoria 16, I-20133 Milano, Italy \\ 3 Physics Department, University of Notre Dame, Notre Dame, Indiana 46556, USA \\ 4 Joint Institute for Nuclear Astrophysics, University of Notre Dame, Notre Dame, Indiana 46556, USA \\ 5 Center for Mathematics and Physics, University of Aizu, Aizu-Wakamatsu, Fukushima 965-8560, Japan \\ 6 RIKEN Nishina Center, Wako 351-0198, Japan
}

Received: date / Revised version: date

\begin{abstract}
The experimental and theoretical studies of Giant Resonances, or more generally of the nuclear collective vibrations, are a well established domain in which sophisticated techniques have been introduced and firm conclusions reached after an effort of several decades. From it, information on the nuclear equation of state can be extracted, albeit not far from usual nuclear densities. In this contribution, which complements other contributions appearing in the current volume, we survey some of the constraints that have been extracted recently concerning the parameters of the nuclear symmetry energy. Isovector modes, in which neutrons and protons are in opposite phase, are a natural source of information and we illustrate the values of symmetry energy around saturation deduced from isovector dipole and isovector quadrupole states. The isotopic dependence of the isoscalar monopole energy has also been suggested to provide a connection to the symmetry energy: relevant theoretical arguments and experimental results are thoroughly discussed. Finally, we consider the case of the charge-exchange spin-dipole excitations in which the sum rule associated with the total strength gives in principle access to the neutron skin and thus, indirectly, to the symmetry energy.
\end{abstract}

PACS. 21.65.Ef Symmetry energy - 24.30.Cz Giant resonances - 21.60.Jz Nuclear Density Functional Theory

\section{Introduction}

As is testified by the variety of contributions in this volume, complementarity of the sources of information is a vital component of our understanding of the symmetry energy. In our contribution, we review several attempts to use the nuclear collective excitations as a tool to infer the properties of the symmetry energy. We stress that in most of these cases, although we can only access densities that are relatively close to the usual nuclear density, the information can be considered as quite accurate due both to well established experimental techniques and to the availability of microscopic methods that have been tested against many other observables. This is at variance with other situations (astrophysical observations and, to some extent, heavy-ion collisions) in which one is potentially able to explore a broader range of densities, but at the expense of facing with more global and specific uncertainties.

We start by reviewing the basic equations related to symmetry energy. For any nuclear system the total energy must depend both on neutron and proton densities $\rho_{n}$ and $\rho_{p}$

$$
E=\int d^{3} r \mathcal{E}\left(\rho_{n}(\boldsymbol{r}), \rho_{p}(\boldsymbol{r})\right),
$$

where $\mathcal{E}$ is the energy density and we have assumed locality for the sake of simplicity. In the following, we will use $q$ as a generic label for neutrons and protons. In finite systems the energy can actually depend not only on the spatial densities, but also on their gradients $\nabla \rho_{q}$, on the kinetic energy densities $\tau_{q}$, as well as on other generalised densities like the spin-orbit densities $J_{q}$; however, in infinite matter, one has a simple expression in terms of the spatial densities only (cf., e.g., Ref. [1]).

Instead of $\rho_{n}$ and $\rho_{p}$, one can use the total density $\rho$ and the local neutron-proton asymmetry,

$$
\beta \equiv \frac{\rho_{n}-\rho_{p}}{\rho} .
$$

In asymmetric matter, we can make a further simplification on $\mathcal{E}(\rho, \beta)$ by making a Taylor expansion in $\beta$ and retaining only the quadratic term (odd powers of $\beta$ are 
obviously forbidden due to isospin symmetry),

$$
\begin{aligned}
\mathcal{E}(\rho, \beta) & \approx \mathcal{E}_{0}(\rho, \beta=0)+\mathcal{E}_{\text {sym }}(\rho) \beta^{2} \\
& =\mathcal{E}_{0}(\rho, \beta=0)+\rho S(\rho) \beta^{2} .
\end{aligned}
$$

The first term on the r.h.s. is the energy density of symmetric nuclear matter $\mathcal{E}_{\mathrm{nm}}$, while the second term defines the main object of all studies in this volume, namely the symmetry energy $S(\rho)$. The symmetry energy at saturation $S\left(\rho_{0}\right)$ is denoted by different symbols in the literature viz. $J, a_{\tau}$ or $a_{4}$; we shall use $J$ in what follows. We stress, however, that Eq. (3) is not really a simplification: the coefficient of the term in $\beta^{4}$ which should follow is negligible in most models at the densities of interest for this work (see, e.g., Ref. 22 and in particular Fig. 1 of that work; this conclusion has been also systematically checked in Ref. 3] with all the models used therein). We remind the reader that the pressure can be written in a uniform system as

$$
P=-\left.\frac{\partial E}{\partial V}\right|_{A}=\left.\rho^{2} \frac{\partial}{\partial \rho} \frac{\mathcal{E}}{\rho}\right|_{A},
$$

where $A$ is the nucleon number. Thus, although it is customary to refer to the energy per particle as the "equation of state", the relationship with the quantity that better fits such name, that is, the pressure as a function of the density, is evident from the latter equation.

The overall trend of the symmetry energy is poorly known, but the main quantities on which attention has been focused are

$$
\begin{aligned}
J & \equiv S\left(\rho_{0}\right), \\
L & \equiv 3 \rho_{0} S^{\prime}\left(\rho_{0}\right), \\
K_{\mathrm{sym}} & \equiv 9 \rho_{0}^{2} S^{\prime \prime}\left(\rho_{0}\right) .
\end{aligned}
$$

$L$ is often referred to as the "slope parameter".

It is expected, therefore, that the isovector giant resonances may be the main source of information for the symmetry energy. They are collective excitations in which most of the nucleons participate, as it is known from the fact that they exhaust a large fraction of the appropriate sum rules. Especially in heavy nuclei we can assume that if an isovector external field displaces the protons with respect to the neutrons, by creating a local proton-neutron asymmetry, the restoring force in the harmonic approximation can be related to

$$
\frac{\delta^{2} E}{\delta \beta^{2}}
$$

if the oscillations involve only variations of $\beta$. Of course such simple argument should be taken with care: the nucleus does not have uniform values of $\rho$ and $\beta$; isospin is not an exact quantum number so that isoscalar and isovector oscillations are not well separated; and, finally, quantum effects like shell structure or pairing may also spoil the simple classical arguments. However, we will show in what follows that these warnings, although manifesting themselves in some error bar that we must attribute to our extractions of the symmetry energy, do not prevent at all deducing values for the symmetry energy around saturation. We shall also show that isoscalar modes like the monopole resonance can be somewhat related to the symmetry energy if one observes this mode along an isotopic chain.

The outline of the paper is the following. In Secs. 2,3 and 4 we discuss some constraints coming from different isovector states, that is, the well-known giant dipole resonance, the so-called pygmy resonances, and the isovector giant quadrupole resonance, respectively. In Sec. 5 we provide theoretical arguments why the isoscalar monopole, when measured in nuclei with neutron excess, could also provide access to some key parameter associated with the symmetry energy; the related experimental data and quantitative conclusions are drawn in Sec. 6. In Sec. 7 we move to the spin-dipole mode excited by charge-exchange reactions, namely to the spin-dipole mode of excitation, whose total strength is related with the neutron skin; we review the experimental findings and the associated theoretical analysis. Finally, we provide a short summary in Sec. 7 .

\section{Symmetry energy from the IVGDR}

The relationship between the symmetry energy and the most collective and well known isovector giant resonance, namely the isovector giant dipole resonance (IVGDR), can be well elucidated by some macroscopic model. As it has been done in Ref. [3], one can start as a guideline from the hydrodynamical model of giant resonances, as proposed by E. Lipparini and S. Stringari 4. They assume an energy functional (1) which is simplified yet sufficiently realistic, solve the macroscopic equations for the densities and currents, and extract expressions for the moments $m_{1}$ and $m_{-1}$ associated with an external operator $F\left(m_{k} \equiv \int d E S(E) E^{k}\right.$ where $S$ is the strength function associated with $F$ ). The expression for $m_{1}$ is proportional to $(1+\kappa)$, where $\kappa$ is the well-known "enhancement factor" which in the case of Skyrme forces is associated with their velocity dependence [1]. The expression for $m_{-1}$, in the case of an isovector external operator, includes integrals involving $\mathcal{E}_{\text {sym }}$ and $F$. They can be evaluated in a simple way if one assumes the validity of the leptodermous expansion. In this way, one introduces volume and surface coefficients of $\mathcal{E}_{\text {sym }}$ that can be denoted by $b_{\mathrm{vol}}$ and $b_{\text {surf }}$, respectively. By specializing $F$ to the isovector dipole case, the following expression is obtained:

$$
E_{-1} \equiv \sqrt{\frac{m_{1}}{m_{-1}}}=\sqrt{\frac{3 \hbar^{2}}{m\left\langle r^{2}\right\rangle} \frac{b_{\mathrm{vol}}}{\left(1+\frac{5}{3} \frac{b_{\mathrm{surf}}}{b_{\mathrm{vol}}} A^{-\frac{1}{3}}\right)}(1+\kappa)} .
$$

This equation has been found to yield values of the centroid energy which are in rather good agreement with those of microscopic Hartree-Fock plus Random Phase Approximation (HF-RPA) calculations performed with microscopic Skyrme interactions 3,5. In fact, the same equation has been used in a previous study [5], in order to constrain directly the parameters of the isovector part of the Skyrme interaction. 
Although there is not a straightforward analytic relation between Eq. (7) and a similar expression that contains the symmetry energy, a semi-analytic relationship has been found in Ref. 3]. The plausibility of such a relation can be briefly discussed here. The coefficient $b_{\mathrm{vol}}$ can be identified with $J$. If the nucleus had a sharp surface this would be the only quantity appearing in Eq. (7). The relevance of the nuclear surface, however, manifests itself in the correction $\left(1+\frac{5}{3} \frac{b_{\text {surf }}}{b_{\mathrm{vol}}} A^{-\frac{1}{3}}\right)^{-1}$. One could then assume that the r.h.s. of Eq. (7) does not scale as $\sqrt{b_{\mathrm{vol}}} \equiv \sqrt{S\left(\rho_{0}\right)}$, but rather as $\sqrt{S(\bar{\rho})}$ where $\bar{\rho}$ is some value of density below the saturation density $\rho_{0}$, namely it is an average density that takes into account the fact that some nucleons are localised in the inner part of the nucleus where the density is $\rho_{0}$ while others are more localised at the surface where the density is lower.

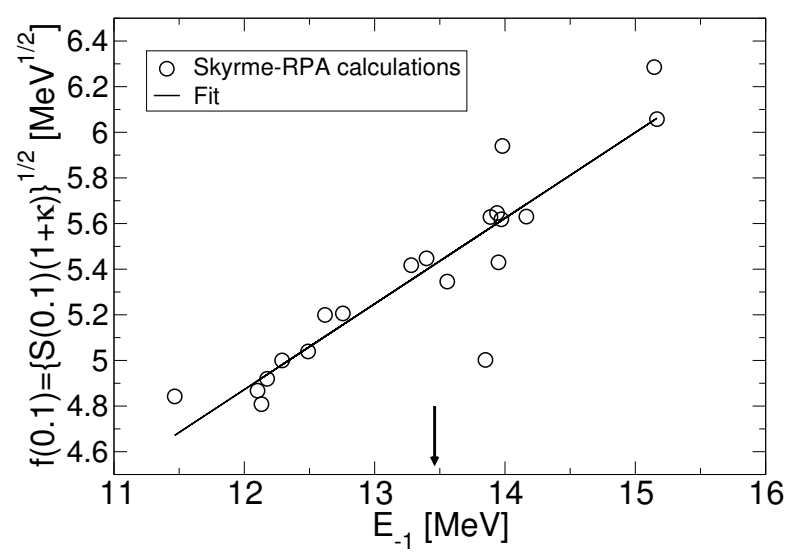

Fig. 1. Correlation associated with Eq. $\sqrt{8}$ in the case of ${ }^{208} \mathrm{~Pb}$. The empty symbols correspond to the microscopic SkyrmeRPA calculations and the line is a linear fit whose correlation coefficient is 0.91. The complete information about the Skyrme models that have been employed, can be found in Ref. [3] from which this figure has been adapted. The arrow indicates the experimental value for $E_{-1}$ from Ref. [6].

In Ref. 3 it has been found indeed that such a correlation between $E_{-1}$ (calculated within HF-RPA) and $\sqrt{S(\bar{\rho})}$ exists. One has to consider the term $(1+\kappa)$, while the value of $\left\langle r^{2}\right\rangle$ does not vary significantly when one changes the model used to calculate the dipole. For heavy nuclei like ${ }^{124} \mathrm{Sn}$ or ${ }^{208} \mathrm{~Pb}$ the value of $\bar{\rho}$ is around $0.1 \mathrm{fm}^{-3}$ whereas this value tends to lower in, e.g., ${ }^{40} \mathrm{Ca}$. The microscopic HF-RPA results for $E_{-1}$ have been calculated using a broad set of Skyrme forces and a strong linear correlation of the type

$$
\sqrt{S(\bar{\rho})(1+\kappa)}=a+b E_{-1}(R P A)
$$

has been found. Typically, the correlation coefficient is around $\approx 0.9$ as it can be seen in Fig. 1 .

Unfortunately, a very precise determination of the enhancement factor $\kappa$ (in other word, of the total dipole EWSR up to high enough energies) is not available. How- ever, by using the case of ${ }^{208} \mathrm{~Pb}$ and inserting the experimental value for the IVGDR in Eq. (8), it has been found that

$$
23.3 \mathrm{MeV}<S(0.1)<24.9 \mathrm{MeV},
$$

where the error takes into account the uncertainty of the linear fit as well as of the experimental value of $\kappa$.

\section{Pygmy Dipole Resonance and other observables related to the dipole spectra}

Recently, much interest has been devoted to the dipole strength below the IVGDR. While in light, neutron-rich halo nuclei this strength may be specially enhanced due to the large transition probability of weakly bound neutrons to continuum states ("threshold effect"), this is not the case in medium-heavy nuclei with neutron excess. These nuclei may be either stable or unstable but the neutron excess gives rise only to a neutron skin and not to a halo. In such systems a possible, peculiar mode of vibration has been proposed, namely the oscillation of the neutrons of the skin with respect to the (essentially $\mathrm{N} \approx \mathrm{Z}$ ) core. The frequency of this mode should be lower than the IVGDR and the name "Pygmy Dipole Resonance" (PDR) has been introduced in the literature.

Experimentally, low-lying dipole strength has been found in several nuclei (see, e.g., Fig. 2 of Ref. [7, and the two review papers [8,9]). To understand the microscopic nature of this strength, namely to establish whether it corresponds to the PDR picture that we have just described, or whether it is a non-collective state, is hard if not impossible especially when measurements are not exclusive ones. Typically the PDR strength may arrive up to a few $\%$ of the dipole EWSR. Among the cases in which this strength appears unambiguously, we mention the nuclei ${ }^{132} \mathrm{Sn}[7$ and ${ }^{68} \mathrm{Ni}[10$.

In Ref. [1], using those two nuclei, another type of correlation between dipole properties and the symmetry energy has been found. This correlation is between the percentage of EWSR exhausted by the PDR and the slope parameter $L$ defined in Eq. (5). This correlation appears clearly, although it is not perfect, when $L$ is plotted against the fraction of EWSR calculated not only by means of Skyrme HF-RPA, but by means of relativistic RPA on top of Relativistic Mean Field (RMF) as well. By using such correlation plot, and the aforementioned experimental data, values of $L$ have been extracted for the two nuclei under study. The separate values of $L$ have been extracted with an error that takes into account experimental errors and uncertaintes in the fits of Fig. 2. The two values overlap and if one assumes one can perform a weighted average for both mean values and uncertainties, one obtains

$$
L=64.8 \pm 15.7 \mathrm{MeV} \text {. }
$$

Within the choice of our models, this implies $J=32.3 \pm 1.3$ $\mathrm{MeV}$. All these points are illustrated in Fig. 2.

In Ref. 11, a careful analysis has also been made concerning the neutron skins. As it has been demonstrated by 

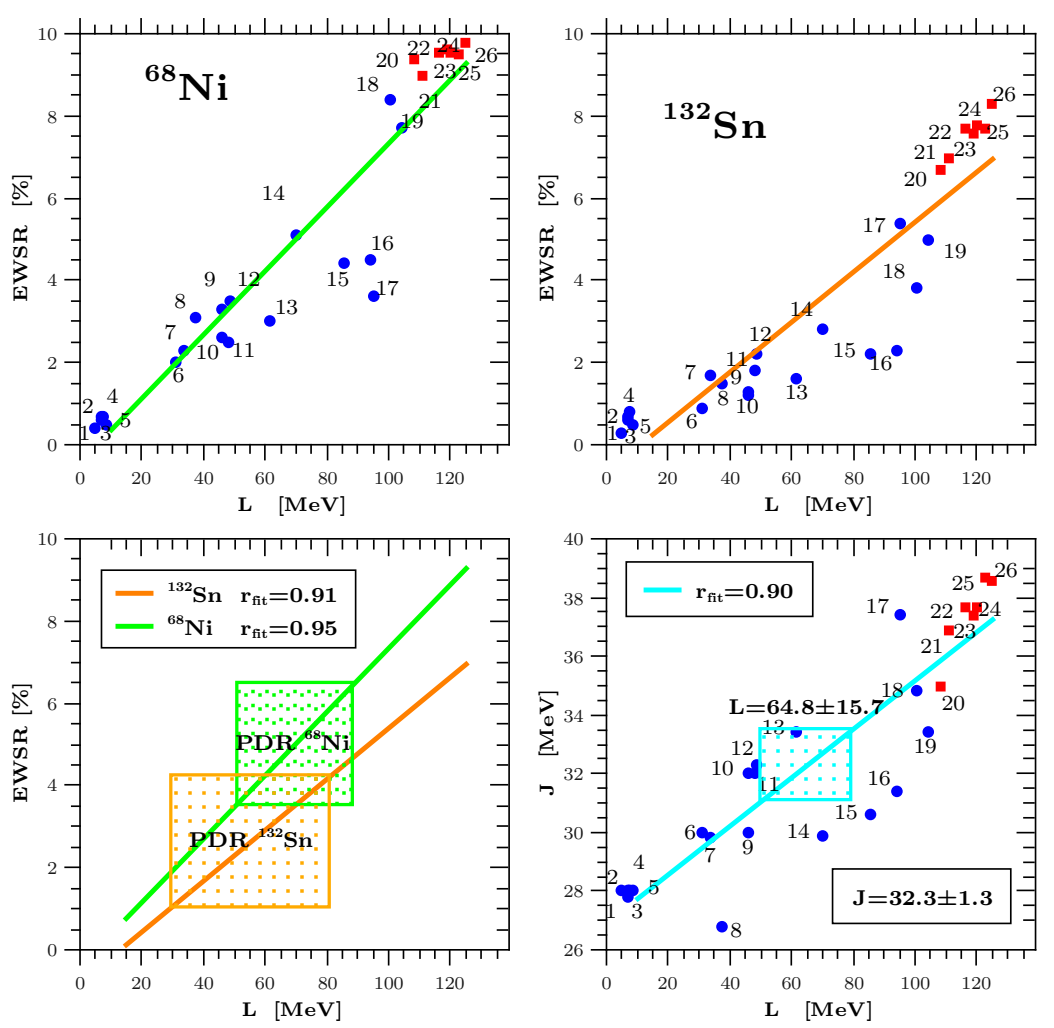

Fig. 2. In the two upper panels the correlation between the slope parameter $L$ and the fraction of EWSR exhausted by the PDR is displayed, for the two nuclei ${ }^{68} \mathrm{Ni}$ and ${ }^{132} \mathrm{Sn}$ respectively. In the lower-left panel the results for the two nuclei are shown together, and the experimental findings are used to deduce and allowed value for $L$ (as it is discussed in the main text). In the lower-right panel a correlation plot $L-J$ is displayed, so that a value for $J$ is also deduced. Taken from Ref. 11] (where the detailed correspondence bwteen numbers and models used can also be found).

more than one author [12,13, the neutron skin is in fact well correlated with $L$ (this can be understood, since if $L$ increases the symmetry energy undergoes larger changes going from the inner part of the nucleus to the surface, and so the system finds it energetically more convenient to localise the excess neutrons on the surface). Consequently, the values of the neutron skins $\Delta R$ extracted from the previous value of $L$ are

$$
\begin{aligned}
\Delta R\left({ }^{68} \mathrm{Ni}\right) & =0.200 \pm 0.015 \mathrm{fm} ; \\
\Delta R\left({ }^{132} \mathrm{Sn}\right) & =0.258 \pm 0.024 \mathrm{fm} ; \\
\Delta R\left({ }^{208} \mathrm{~Pb}\right) & =0.194 \pm 0.024 \mathrm{fm} .
\end{aligned}
$$

We do not dispose of a model that can help as a guideline to understand in detail the correlation between the EWSR of the PDR and $L$. In a simplified picture, if the PDR is really a mode in which the excess neutrons participate and their dynamics is decoupled from the IVGDR, then the correlation we have discussed can be intuitively understood. In fact, the percentage of EWSR of the PDR increases with the number of neutrons belonging to the "skin" 15, and the skin increases with $L$ as we have just discussed.

However, this picture may not be valid in all nuclei where the PDR shows up. In Ref. [16, it has been claimed that the PDR has a single-particle character: this analysis has been carried out using a specific local energy functional, namely SV-bas. In Ref. [17 the microscopic character of the PDR has been analysed in detail in the nuclei ${ }^{68} \mathrm{Ni},{ }^{132} \mathrm{Sn}$ and ${ }^{208} \mathrm{~Pb}$ : while for certain Skyrme energy functionals (those characterised by a larger value of $L$ ) the PDR shows up as a well-defined peak so that it can be truly defined as a resonance, in other cases this does not happen. Consequently, it may be more cautious to refer to the low-lying dipole strength as composed of "pygmy dipole states" (PDSs). These states have also a mixed isospin character and in the isoscalar response they show up more clearly, and display more coherence of the microscopic particle-hole ( $\mathrm{p}-\mathrm{h})$ amplitudes, than in the isovector response. A similar analysis, leading to consistent conclusions, has been performed in Ref. [18. A possible component of toroidal motion in the low-energy dipole strength had been initially claimed in the microscopic calculations of Ref. 19] and recently revived (see, e.g., 20] and references therein). About the nature of the pygmy states, and the question whether any relation with the symmetry energy or the neutron skin has to be expected, the reader can also consult the contribution by J. Piekarewicz to this volume (and references therein), as well as the works on the PDR by T. Inakura and co-workers [21,22].

In conclusion, the nature of the PDR is still strongly debated because of its complex and mixed (isoscalar/isovector, 
surface/volume, irrotational/toroidal) character. Certainly, more experimental and theoretical investigation should be envisaged. For the time being, it remains intriguing how despite all warnings the EWSR carried by the PDR could lead to very reasonable values of $J$ and $L$ as it has been shown in the first part of this Section.

We do not discuss in this contribution other aspects related to IV dipole observables and the symmetry energy. The relevance of the total dipole polarizability [23], and the fact that $L$ turns out to be correlated with the product of $J$ times the dipole polarizability 24, are thoroughly discussed in the contribution to this volume by J. Piekarewicz.

\section{Symmetry energy from the IVGQR}

The properties of the isovector giant quadrupole resonance (IVGQR) have not been determined so accurately for some time, due to the lack of experimental probes having good selectivity. Recently, at the $\mathrm{HI} \gamma \mathrm{S}$ facility, it has been demonstrated that the scattering of polarized photons can provide a direct measurement of the IVGQR properties, without the uncertainties associated with hadronic probe experiments [25]. This experimental achievement has motivated the attempt to extract information about the symmetry energy.

The energy of the IVGQR receives contribution from the unperturbed $\mathrm{p}-\mathrm{h}$ configurations at $2 \hbar \omega$ excitation energy, plus some correlation energy related to the residual interaction. Since the residual interaction is in the isovector channel, it can be naturally linked with the symmetry energy. In Ref. 26] the quantum harmonic oscillator model has been applied to the IVGQR case and, with mild assumptions and taking care of the fact that the unperturbed energy can be related to the effective mass and, in turn, to the isoscalar GQR energy, the main result is the following formula:

$$
E_{\mathrm{IVGQR}} \approx 2\left[\frac{\left(E_{\mathrm{ISGQR}}\right)^{2}}{2}+2 \frac{\varepsilon_{\mathrm{F}_{\infty}}^{2}}{A^{2 / 3}}\left(\frac{3 S\left(\rho_{A}\right)}{\varepsilon_{\mathrm{F}_{\infty}}}-1\right)\right]^{1 / 2},
$$

where $\varepsilon_{\mathrm{F}_{\infty}}$ is is the Fermi energy for symmetric nuclear matter at saturation density, and $S\left(\rho_{A}\right)$ is the symmetry energy at some average nuclear density for the nucleus having mass number A. As we have done above in the IVGDR case, we can choose this average density as 0.1 $\mathrm{fm}^{-3}$. A first important outcome of the previous equation is that the same value of $S(0.1)$ that we have derived in Eq. (9) is consistent with the experimental energies of the ISGQR and IVGQR, and turns out to be further validated by this fact.

Both Skyrme and relativistic mean-field models do indeed follow quite well the scaling predicted by Eq. $(12)$. In the case of the Skyrme models, we demonstrate this fact in Fig. 3. The models have been built by using the same protocol of the recent parameter set SAMi 27. In some of them all nuclear matter parameters have been kept fixed as in the original force, but the effective mass has been
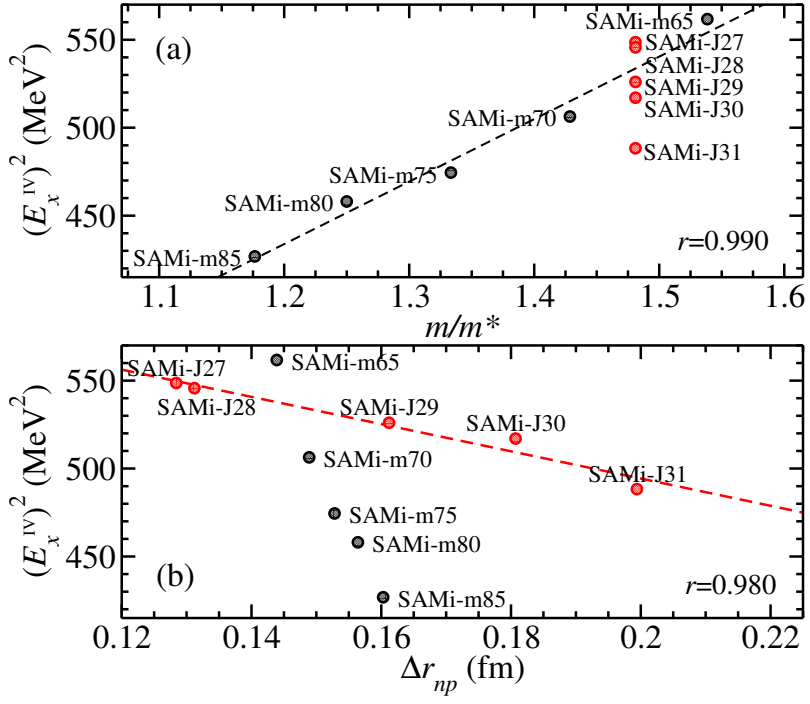

Fig. 3. Sensitivity of the energy of the IVGQR in ${ }^{208} \mathrm{~Pb}$ to the effective mass (upper panel) and to the neutron skin (lower panel). For details about the models whose names appear in the panels, and for an explanation of the emerging correlations, see the main text. Taken from Ref. [26].

changed: thus, SAMi-m85 means that $m * / m$ is 0.85 ; in others, all nuclear matter properties have been kept fixed but the symmetry energy at saturation has been changed: in this case, SAMi-J27 means that $J=27 \mathrm{MeV}$.

From the upper panel of Fig. 3 it is clear that if the symmetry energy properties are kept fixed, the energy increases if the square root of the effective mass decreases. This is due to the first term of Eq. 12 , as the ISGQR energy is known to scale with $\sqrt{m / m^{*}}[28$. In the same way, it is expected that the second term in Eq. (12) plays a role and produces an increase of the IVGQR energy with $S(0.1)$. Actually in our fitting protocol we fix $J$, and for increasing values of this quantity the result of the fit is a comparatively more important decrease at the same time of $L$ and $S(0.1)$ : therefore, both the neutron skin and the IVGQR energy decrease, displaying consequently the interesting correlation of the lower panel of Fig. 3. If we use such kind of correlations between the IVGQR energy and the properties of the symmetry energy, assuming a value of $J=32 \pm 1 \mathrm{MeV}$ we extract

$$
\begin{aligned}
L & =37 \pm 18 \mathrm{MeV} ; \\
\Delta R\left({ }^{208} \mathrm{~Pb}\right) & =0.14 \pm 0.03 \mathrm{fm} .
\end{aligned}
$$

Note that these values are lower but still compatible with the results $(10)$ and $(11)$ extracted from the PDR.

\section{Nuclear Incompressibility and symmetry energy parameters}

The incompressibility $K_{\infty}$ of infinite symmetric matter is defined by the second derivative of the energy per particle $\mathcal{E}_{0}(\rho, \beta=0) / \rho$ with respect to the density $\rho$ at the 
saturation point,

$$
K_{\infty}=\left.9 \rho^{2} \frac{d^{2}}{d \rho^{2}}\left(\frac{\mathcal{E}_{0}(\rho, \beta=0)}{\rho}\right)\right|_{\rho=\rho_{0}},
$$

where $\mathcal{E}_{0}(\rho, \beta=0)$ is the isoscalar part of the energy density $\mathcal{E}(\rho, \beta)$ for nuclear matter given in Eq. (3). The nuclear matter incompressibility $K_{\infty}$ is not a directly measurable quantity. Instead, the energy of isoscalar giant monopole resonance, $E_{I S G M R}$, is expressed in terms of the finite nucleus incompressibility $K_{A}$ as 29,30

$$
E_{I S G M R}=\sqrt{\frac{\hbar^{2} K_{A}}{m<r^{2}>_{m}}},
$$

where $m$ is the nucleon mass and $\left\langle r^{2}\right\rangle_{m}$ is the mean square mass radius of the ground state. The finite nucleus incompressibility can be parameterized by means of a similar expansion as the liquid drop mass formula with the volume, surface, symmetry and Coulomb terms [28]:

$$
K_{A}=K_{v o l}+K_{\text {surf }} A^{-1 / 3}+K_{\tau} \delta^{2}+K_{C o u l} \frac{Z^{2}}{A^{4 / 3}},
$$

where $\delta=(N-Z) / A$. We denote by $K_{\tau}$ the asymmetry term of the finite nucleus incompressibility $K_{A}$ because the symbol $K_{\text {sym }}$ has been already used as one of the isovector nuclear matter properties in Eq. (5). The volume term $K_{v o l}$ of the finite nucleus incompressibility $K_{A}$ is directly related to the nuclear matter incompressibility $K_{\infty}$. The asymmetry term $K_{\tau}$ is related to nuclear matter properties as 28, 31

$$
K_{\tau}=K_{\text {sym }}+3 L-L B,
$$

where $B$ is proportional to the third derivative of the energy density with respect to the density at the saturation point,

$$
B=\left.\frac{27 \rho_{0}^{2}}{K_{\infty}} \frac{d^{3} \mathcal{E}_{0}(\rho, \beta=0)}{d \rho^{3}}\right|_{\rho=\rho_{0}} .
$$

In Refs. $32,33, K_{\tau}$ is expressed in terms of the skewness parameter $Q$ as

$$
K_{\tau}=K_{s y m}-6 L-\frac{L Q}{K_{\infty}}
$$

where $Q$ is defined as the third derivative of the energy per nucleon with respect to the density at the saturation point,

$$
Q=\left.27 \rho_{0}^{2} \frac{d^{3}\left(\mathcal{E}_{0}(\rho, \beta=0) / \rho\right)}{d \rho^{3}}\right|_{\rho=\rho_{0}} .
$$

The formulas (18) and 20 look different, but they are equivalent since $B$ and $Q$ are related by the equation

$$
B=9+\frac{Q}{K_{\infty}}
$$

The analytic formulas for $K_{\text {surf }}$ and $K_{\text {Coul }}$ are given by

$$
\begin{gathered}
K_{\text {surf }}=4 \pi r_{0}^{2}\left[4 \sigma\left(\rho_{0}\right)+\left.9 \rho_{0} \frac{d^{2} \sigma}{d \rho^{2}}\right|_{\rho=\rho_{0}}+2 \sigma\left(\rho_{0}\right) B\right] \\
K_{\text {Coul }}=\frac{3}{5} \frac{e^{2}}{r_{0}}(1-B)
\end{gathered}
$$

where $r_{0}$ is the radius constant defined by

$$
r_{0}=\left(\frac{3}{4 \pi \rho_{0}}\right)^{1 / 3} \text {. }
$$

In Eq. (23), $\sigma$ is the surface tension in symmetric semiinfinite nuclear matter defined by

$$
\sigma\left(\rho_{0}\right)=\int_{-\infty}^{\infty}\left[\mathcal{E}(\rho, \beta=0)-\frac{\mathcal{E}_{0}\left(\rho_{0}, \beta=0\right)}{\rho_{0}} \rho\right] d z .
$$

$K_{\text {surf }}$ can be evaluated by the extended Thomas-Fermi approximation and the scaled HF calculations of semiinfinite nuclear matter in the Skyrme Hartree-Fock (SHF) model. These evaluations show that the approximate relation $K_{\text {surf }} \sim-K_{\infty}$ holds within an accuracy of a few $\%$ in the SHF model. In relativistic models, the extended Thomas-Fermi approximation gives as a result a slightly larger surface contribution, for example, $K_{\text {surf }} \sim-1.16 K_{\infty}$ in the case of NL3.

It is feasible to calculate the values of $K_{\tau}$ and $K_{C o u l}$ by using various Skyrme Hamiltonians and relativistic Lagrangians. It was pointed out in Ref. 34 that there are no clear correlations between $L$ and $K_{\infty}$, and between $K_{\text {sym }}$ and $K_{\infty}$. On the other hand it is remarkable that $K_{\tau}$, as a linear combination of $K_{\text {sym }}, L$ and $B$, show a clear correlation with $K_{\infty}$ having a large correlation coefficient, especially in SHF models 34. Correlation plots between $K_{\tau}$, the symmetry coefficient of the nuclear incompressibility, and the parameters $J, L$ and $K_{\text {sym }}$ characterising the symmetry energy, are displayed in Figs. 4 , 5 and 6 , respectively [35]. The plot involving $J$ and $K_{\tau}$ in Fig. 4 shows a clear correlation with a negative slope. We can see also a similar correlation between $L$ and $K_{\tau}$ in Fig. 5. Both correlation coefficents are between -0.6 and -0.7 . Thus, empirical information on $K_{\tau}$ from e.g. the isotopic dependence of the ISGMR energies may give some constraint on these two values. We will come back to this point at the end of Sec. 6. On the other hand, the points in the plot of $K_{s y m}$ vs. $K_{\tau}$ are rather scattered, and it looks difficult to constrain the value of $K_{s y m}$ from the empirical value of $K_{\tau}$. We should also make the remark that the results of RMF and Skyrme models have rather large overlap and give consistent results in the correlation plots of Figs. 4 and 5 .

It was pointed out that any Hamiltonian which has a larger $K_{\infty}$ gives a smaller $K_{\tau}$ [31. The variations of $K_{\tau}$ for the Skyrme interactions are

$$
K_{\tau}=(-400 \pm 100) \mathrm{MeV} \quad \text { for Skyrme interactions. }
$$




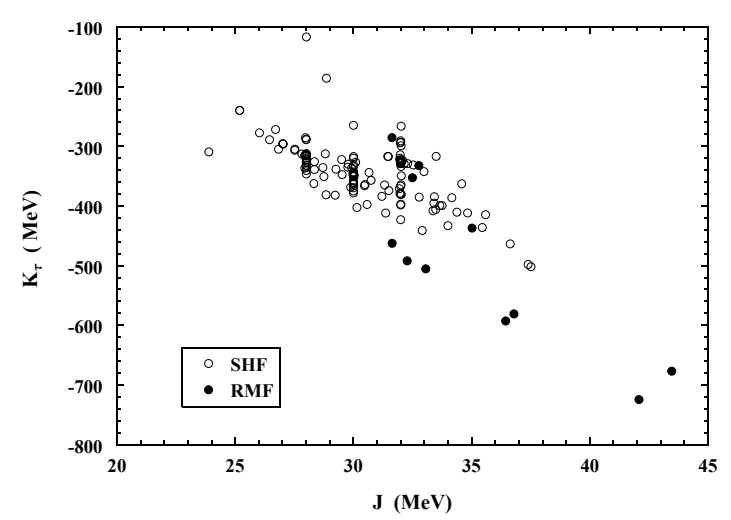

Fig. 4. Correlation between the asymmetry term of the finite nucleus incompressibility $K_{\tau}$ and the volume symmetry energy $J$, calculated by using various Skyrme parameter sets (SHF, open circles) and relativistic Lagrangians (RMF, filled circles).

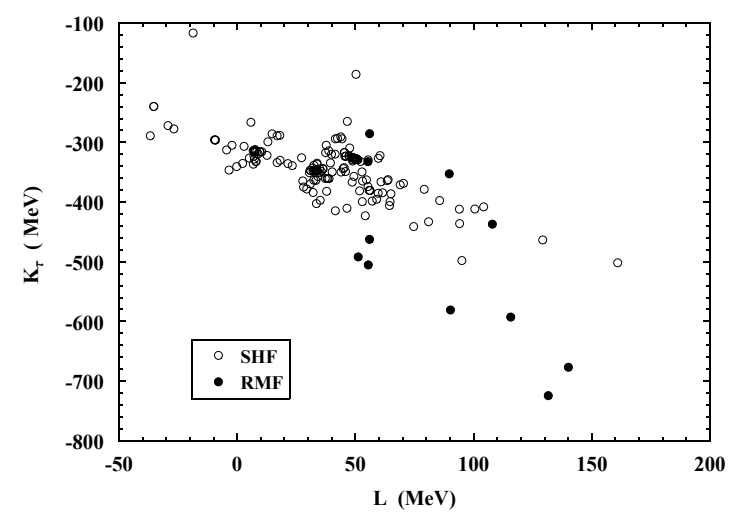

Fig. 5. Correlation between the asymmetry term of the finite nucleus incompressibility $K_{\tau}$ and the slope parameter $L$, calculated by using various Skyrme parameter sets (SHF, open circles) and relativistic Lagrangians (RMF, filled circles).

On the other hand, the values of RMF are largely negative and have more variation among the seven effective relativistic mean field (RMF) Lagrangians,

$$
K_{\tau}=(-620 \pm 180) \mathrm{MeV} \quad \text { for RMF Lagrangians. }
$$

In principle, the value of $K_{\text {Coul }}$ should be model-independent. Among the 13 parameter sets of Skyrme interactions, the variation of $K_{\text {Coul }}$ is rather small,

$$
K_{\text {Coul }}=(-5.2 \pm 0.7) \mathrm{MeV}
$$

compared with that of $K_{\tau}$. The values of $K_{\text {Coul }}$ in RMF show essentially the same trend, but have a larger variation.

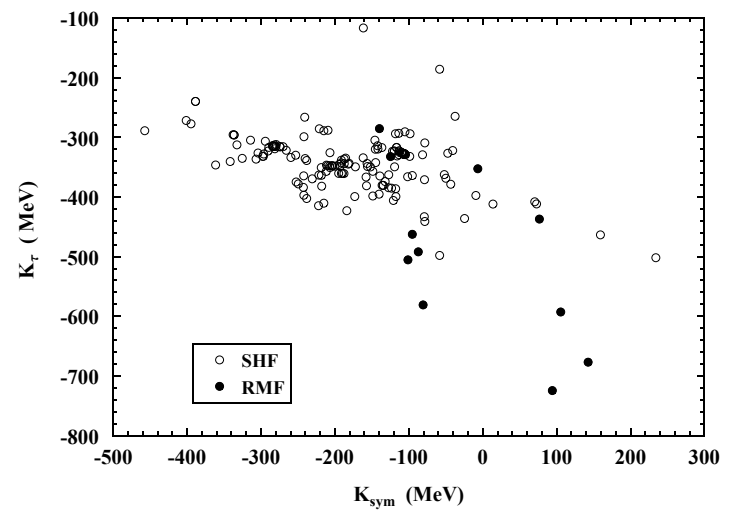

Fig. 6. Correlation between the asymmetry term of the finite nucleus incompressibility $K_{\tau}$ and the second derivative of the symmetry energy $K_{\text {sym }}$, calculated by using various Skyrme parameter sets (SHF, open circles) and relativistic Lagrangians (RMF, filled circles).

\section{Nuclear incompressibility and the asymmetry term from the ISGMR}

The study of the isoscalar giant monopole resonance (ISGMR) provides a direct experimental connection to nuclear incompressibility in finite nuclear systems. The centroid energy of ISGMR, $E_{I S G M R}$, can be related to the nuclear incompressibility of finite nuclear matter, $K_{A}$, as given by Eq. (16). The ISGMR strength distribution can be determined experimentally via inelastic scattering of isoscalar probes. The most commonly-used, and effective, probe for such investigations has been the $\alpha$-particle (the ${ }^{4} \mathrm{He}$ nucleus). In these investigations, inelastic scattering measurements are performed off a particular target at very forward angles, including $0^{\circ}$.

The importance of making measurements at such extreme forward angles, including $0^{\circ}$, is twofold: the cross section for the ISGMR peaks at $0^{\circ}$, and the $L=0$ angular distribution is most distinct at the very forward angles. These measurements are, however, extremely difficult since the primary beam passes very close to the scattered particles at these angles and one requires a combination of a high-quality, halo-free beam, and an appropriate magnetic spectrometer. The high-resolution magnetic spectrometer Grand Raiden, at the Research Center for Nuclear Physics (RCNP) at Osaka University, Japan 36. is a most suitable such instrument; similar measurements are being carried out at the Texas A \& M University cyclotron facility as well 37. An unmatched asset of Grand Raiden is that its optical properties allow for collection of inelastic scattering spectra practically free of all instrumental background that had been a bane of such measurements in the past.

The inelastic scattering spectra are analyzed using multipole decomposition analysis (MDA) [38,39] to extract the ISGMR strength distributions, the centroid of which 
can give the compressibility, $K_{A}$ of the nucleus under investigation. Examples of such "background-free", spectra, as well as the details of the experimental techniques and analysis procedures for these measurements have been provided in several recent reports from the RCNP work (see, for example, Refs. [40,41,33]).

To go from $K_{A}$ to $K_{\infty}$, one builds a class of energy functionals, $E(\rho)$ [cf. Eq. (1)], with different parameters that allow calculations for nuclear matter and finite nuclei in the same theoretical framework. The parameterset for a given class of energy functionals is characterized by a specific value of $K_{\infty}$. The ISGMR strength distributions are obtained for different energy functionals in a self-consistent RPA calculation. The $K_{\infty}$ associated with the interaction that best reproduces the ISGMR centroid energies is, then, considered the correct value [28].

Following this procedure, both relativistic and nonrelativistic calculations give $K_{\infty}=240 \pm 20 \mathrm{MeV}$,42,43 44, 45, 46. These accurately calibrated relativistic and nonrelativistic models reproduce very well the ISGMR centroid energies in the "standard" nuclei, ${ }^{90} \mathrm{Zr}$ and ${ }^{208} \mathrm{~Pb}$. However, it has been established in recent measurements on the $\mathrm{Sn}$ and $\mathrm{Cd}$ isotopes 41,33 that this value of $K_{\infty}$ significantly overestimates $E_{I S G M R}$ for these "open shell" nuclei. In other words, it would appear that the $\mathrm{Sn}$ and Cd nuclei are "softer", considering the $E_{I S G M R}$ from just these nuclei would yield an appreciably lower value for $K_{\infty}$. Pairing correlations have been suggested as a reason for this softening; yet, the results are not conclusive 47 48. 49 .

As noted in the previous Section, $K_{A}$ may be parameterized as:

$K_{A} \approx K_{\text {vol }}\left(1+c A^{-1 / 3}\right)+K_{\tau}\left(\frac{N-Z}{A}\right)^{2}+K_{\text {Coul }} \frac{Z^{2}}{A^{4 / 3}}$.

Here, $c \approx-1$ as noted previously and discussed in detail in Ref. 50; $K_{\text {Coul }}$ is essentially a model-independent term (in the sense that the deviations from one theoretical model to another are quite small) [31] and $K_{\tau}$ is the asymmetry term. Although closely related, the finitenucleus asymmetry term $K_{\tau}$ should not be confused with the corresponding term in infinite nuclear matter-a quantity also denoted by $K_{\tau}$ at times, but which should actually be written as $K_{\tau}^{\infty}$ (we have introduced this quantity in Eq. (18) above, and showed that it should not be confused with $K_{\text {sym }}$ either; in fact, the asymmetry coefficient of the finite nucleus incompressibility does not take contribution merely from the second derivative of the symmetry energy). $K_{\tau}^{\infty}$ should never be regarded as the $A \rightarrow \infty$ limit of the finite-nucleus asymmetry $K_{\tau}$. Yet the fact that $K_{\tau}$ is both experimentally accessible and strongly correlated with $K_{\tau}^{\infty}$ is vital in placing stringent constraints on the density dependence of the symmetry energy. It is the strong sensitivity of $K_{\tau}^{\infty}$ to the density dependence of the symmetry energy that makes this investigation of critical importance in constraining the EOS of neutron-rich matter.
This asymmetry term, $K_{\tau}$, can be obtained by investigating the ISGMR over a series of isotopes for which the neutron-proton asymmetry, $(N-Z) / A$, changes by an appreciable amount. Coming back to Eq. (30), for a series of isotopes, the difference $K_{A}-K_{\text {Coul }} Z^{2} A^{-4 / 3}$ may be approximated to have a quadratic relationship with the asymmetry parameter $((\mathrm{N}-\mathrm{Z}) / \mathrm{A}))$, of the type $\mathrm{y}=\mathrm{A}$ $+\mathrm{Bx}^{2}$, with $K_{\tau}$ being the coefficient, B, of the quadratic term.

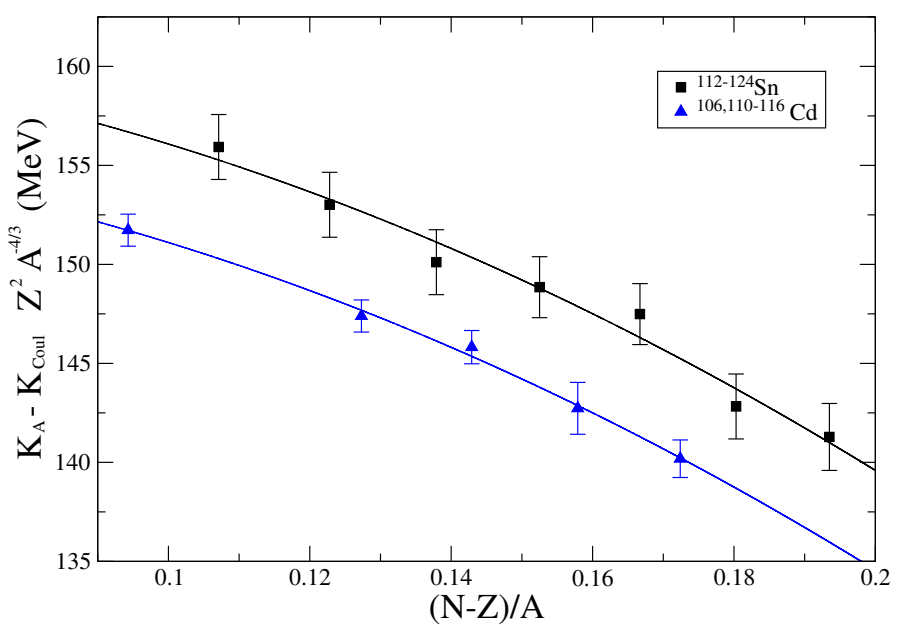

Fig. 7. The difference $K_{A}-K_{C o u l} Z^{2} A^{-4 / 3}$ in the $\mathrm{Sn}$ and $\mathrm{Cd}$ isotopes plotted as a function of the asymmetry parameter, $(N-Z) / A$. The data are from Refs. 41,33. The values of $K_{A}$ have been derived using the customary moment ratio $\sqrt{m_{1} / m_{-1}}$ for the energy of ISGMR, and a value of $5.2 \pm 0.7$ $\mathrm{MeV}$ has been used for $K_{\text {Coul }}$ (see previous Section). The solid lines correspond to $K_{\tau}=-550 \mathrm{MeV}$.

Such an investigation was carried out by Li et al. over the even- $A{ }^{112-124} \mathrm{Sn}$ isotopes [40,41] and by Patel et al. over the even- $A{ }^{106,110-116} \mathrm{Cd}$ isotopes [33]. The $\mathrm{Sn}$ isotopes yielded a value of $K_{\tau}=-550 \pm 100 \mathrm{MeV}$, the $\mathrm{Cd}$ isotopes resulted in $K_{\tau}=-555 \pm 75 \mathrm{MeV}$. Not only are the two values thus obtained in excellent agreement with each other, but also are consistent with values indirectly obtained from several other measurements: $K_{\tau}=-370 \pm 120$ $\mathrm{MeV}$ obtained from the analysis of the isotopic transport ratios in medium-energy heavy-ion reactions 32, $K_{\tau}=-500_{-100}^{+120} \mathrm{MeV}$ obtained from constraints placed by neutron-skin data from anti-protonic atoms across the mass table [51]; and, $K_{\tau}=-500 \pm 50 \mathrm{MeV}$ obtained from theoretical calculations using different Skyrme interactions and relativistic mean-field (RMF) Lagrangians [31. In Fig. 7), we show the data for the $\mathrm{Sn}$ and $\mathrm{Cd}$ isotopes from Refs. 41,33 along with quadratic fits with a common value of $K_{\tau}=-550 \mathrm{MeV}$.

From the correlation plots in Figs. 4 and 5 , one may extract the symmetry energy coefficients $J$ and $L$ from the empirical value $K_{\tau}=-550 \mathrm{MeV}$. We must take into account the error on this latter quantity $( \pm 100 \mathrm{MeV})$, as well as the uncertainties on the linear fits. In this way, $J$ is found to lie in the range $27.7-35.6 \mathrm{MeV}$. On the other 


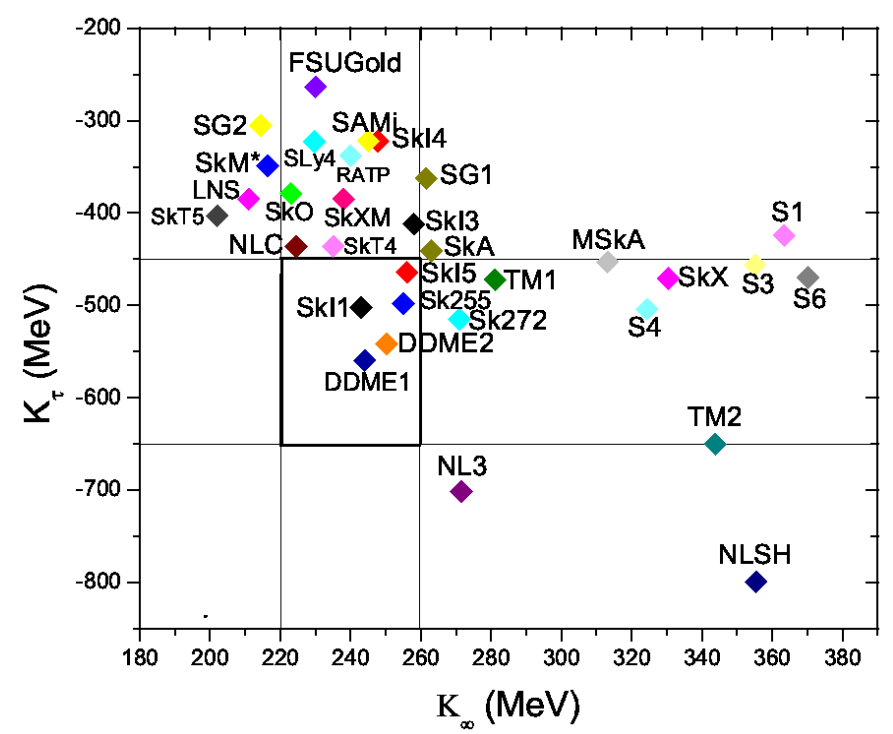

Fig. 8. Values of $K_{\infty}$ and $K_{\tau}$ calculated from the parameter sets of various interactions as labeled 31. The vertical and horizontal lines indicate the experimental ranges of $\mathrm{K}_{\infty}$ and $\mathrm{K}_{\tau}$, as determined from the GMR work.

hand, the correlation between $K_{\tau}$ and $L$ is weaker and we cannot get a meaningful constraint on $L$.

The "experimental" values thus obtained from the ISGMR for $K_{\infty}$ and $K_{\tau}$ taken together can provide a means of selecting the most appropriate of the interactions used in EOS calculations. In Fig. 8, we plot the $K_{\infty}$ and $K_{\tau}$ for a number of interactions used in nuclear structure and EOS calculations. It would appear, indeed, that a vast majority of the interactions fail to meet the criterion established by these measurements. A caveat to this statement, though: the $K_{\tau}$ obtained in these measurements is only an "average" value, and the data cannot disentangle the volume symmetry from higher-order effects like the surface symmetry. Thus, this average value has been identified with the volume symmetry only, and compared with the volume symmetry coefficient provided by the models. It is possible, then, to execute similar fits including higherorder terms and obtain very different values for $K_{\tau}$ [52]; however, the "appropriateness" of the values of the extra terms thus obtained remains unclear.

\section{Spin-dipole resonances and neutron skin}

As was mentioned in Section 3, the neutron skin gives an important information about the constraints on the symmetry energy. It is known that the model-independent non energy-weighted sum rule of charge exchange spin-dipole (SD) excitations is directly related to the neutron skin thickness [53. Recently, SD excitations were studied in ${ }^{90} \mathrm{Zr}$ by the charge-exchange reactions ${ }^{90} \mathrm{Zr}(\mathrm{p}, \mathrm{n}){ }^{90} \mathrm{Nb}[54$ and ${ }^{90} \mathrm{Zr}(\mathrm{n}, \mathrm{p})^{90} \mathrm{Y}$ [55, and the model-independent sum rule for the SD excitations were extracted in Ref. [56] by using multipole decomposition analysis (MDA) [57. The charge exchange reactions $\left({ }^{3} \mathrm{He}, t\right)$ on $\mathrm{Sn}$ isotopes were also studied to extract the neutron skin thickness [58. However, one needs the counter experiment $\left(t,{ }^{3} \mathrm{He}\right)$ or $(\mathrm{n}, \mathrm{p})$ on $\mathrm{Sn}$ isotopes in order to extract the model-independent sum rule value from experimental data. This counter experiment is missing in the case of $\mathrm{Sn}$ isotopes.

The operators for $\lambda$-pole SD transitions are defined as

$$
\hat{S}_{ \pm}^{\lambda}=\sum_{i} t_{ \pm}^{i} r_{i}\left[\sigma \otimes Y_{l=1}\left(\hat{r}_{i}\right)\right]^{\lambda=0,1,2}
$$

with the isospin operators being denoted as $t_{ \pm}=t_{x} \pm$ $i t_{y}$. The model-independent sum rule for the $\lambda$-pole SD operator $\hat{S}_{ \pm}^{\lambda}$ can be obtained as

$$
\begin{aligned}
& S_{-}^{\lambda}-S_{+}^{\lambda}=\sum_{i \in \text { all }}\left|\left\langle i\left|\hat{S}_{-}^{\lambda}\right| 0\right\rangle\right|^{2}-\sum_{i \in \text { all }}\left|\left\langle i\left|\hat{S}_{+}^{\lambda}\right| 0\right\rangle\right|^{2} \\
& =\left\langle 0\left|\left[\hat{S}_{-}^{\lambda}, \hat{S}_{+}^{\lambda}\right]\right| 0\right\rangle=\frac{(2 \lambda+1)}{4 \pi}\left(N\left\langle r^{2}\right\rangle_{n}-Z\left\langle r^{2}\right\rangle_{p}\right) .(32)
\end{aligned}
$$

The sum rule for the spin-dipole operator 31 then becomes

$$
S_{-}-S_{+}=\sum_{\lambda}\left(S_{-}^{\lambda}-S_{+}^{\lambda}\right)=\frac{9}{4 \pi}\left(N\left\langle r^{2}\right\rangle_{n}-Z\left\langle r^{2}\right\rangle_{p}\right)
$$

It should be noted that the sum rule $(33)$ is directly related to the difference between the mean square radius of neutrons and protons with the weight of neutron and proton numbers.

Let us now discuss the integrated SD strength. The integrated SD strength

$$
m_{0}\left(E_{x}\right)=\sum_{\lambda^{\pi}=0^{-}, 1^{-}, 2^{-}} \int_{0}^{E_{x}} \frac{d B\left(\lambda^{\pi}\right)}{d E^{\prime}} d E^{\prime}
$$

is plotted as a function of the excitation energy $E_{x}$ in Fig. 9 for the operators $\hat{S}_{-}^{\lambda}$ and $\hat{S}_{+}^{\lambda}$ in Eq. 31. The value $S_{-}$ is obtained by integrating up to $E_{x}=50 \mathrm{MeV}$ from the ground state of the daughter nucleus ${ }^{90} \mathrm{Nb}\left(E_{x}=57 \mathrm{MeV}\right.$ from the ground state of the parent nucleus ${ }^{90} \mathrm{Zr}$ ), while the corresponding value $S_{+}$is evaluated up to $E_{x}=26$ $\mathrm{MeV}$ from the ground state of ${ }^{90} \mathrm{Y}\left(E_{x}=27.5 \mathrm{MeV}\right.$ from the ground state of ${ }^{90} \mathrm{Zr}$ ). This difference between the two maximum energies of the integrals stems from the isospin difference between the ground states of the daughter nuclei, i.e., $\mathrm{T}=4$ in ${ }^{90} \mathrm{Nb}$ and $\mathrm{T}=6$ in ${ }^{90} \mathrm{Y}$. That is, the 23.6 $\mathrm{MeV}$ difference originates from the difference in excitation energy between the $\mathrm{T}=6$ Gamow-Teller states in the $(\mathrm{p}, \mathrm{n})$ and $(\mathrm{n}, \mathrm{p})$ channels [56]. For both the $S_{-}$and $S_{+}$strength, the calculated results overshoot the experimental data in the energy range $E_{x}=20-40 \mathrm{MeV}$. These results suggest a quenching of $30-40 \%$ of the calculated strength around the peak region. However, the integrated cross sections up to $E_{x}=56 \mathrm{MeV}$ in Fig. 9 approach the calculated values for both the $t_{-}$and $t_{+}$channels.

The $\Delta S=S_{-}-S_{+}$value is shown as a function of $E_{x}$ in the lower panel of Fig. 9. We note that the $\Delta S$ value saturates both in the calculated and the experimental values 
Table 1. Sum rule values of charge exchange SD excitations in $\mathrm{A}=90$ nuclei obtained by the HF+RPA calculations $[59$ ] for ${ }^{90} \mathrm{Nb}$ and $\mathrm{S}_{+}$for ${ }^{90} \mathrm{Y}$ ). The $\mathrm{SD}$ strength is integrated up to $E_{x}=50 \mathrm{MeV}$ for $\mathrm{S}_{-}$and $E_{x}=26 \mathrm{MeV}$ for $\mathrm{S}_{+}$, respectively. The experimental data are taken from Ref. [56. The SD sum rules are given in units of $\mathrm{fm}^{2}$. See the text for details.

\begin{tabular}{l||c|c|c||c|c|c||c|c|c||c|c|c}
\hline & \multicolumn{3}{|c||}{ SIII } & \multicolumn{3}{c||}{ SGII } & \multicolumn{3}{c||}{ SkI3 } & \multicolumn{3}{c}{ SLy4 } \\
\hline$\lambda^{\pi}$ & $S_{-}$ & $S_{+}$ & $\Delta S$ & $S_{-}$ & $S_{+}$ & $\Delta S$ & $S_{-}$ & $S_{+}$ & $\Delta S$ & $S_{-}$ & $S_{+}$ & $\Delta S$ \\
\hline $0^{-}$ & 34.8 & 18.5 & 16.4 & 33.2 & 17.4 & 15.8 & 36.6 & 19.1 & 17.5 & 37.8 & 21.4 & 16.4 \\
\hline $1^{-}$ & 120.8 & 71.7 & 49.1 & 122.0 & 74.3 & 47.7 & 120.8 & 68.2 & 52.7 & 115.8 & 66.4 & 49.4 \\
\hline $2^{-}$ & 130.1 & 48.5 & 81.6 & 125.5 & 45.9 & 79.5 & 139.0 & 51.1 & 87.9 & 138.7 & 56.4 & 82.3 \\
\hline sum & 285.7 & 138.6 & 147.1 & 280.7 & 137.6 & 143.1 & 296.3 & 138.3 & 158.0 & 292.3 & 144.2 & 148.1 \\
\hline \hline $\exp$ & \multicolumn{3}{c|}{$S_{-}=271 \pm 14$} & \multicolumn{4}{c|}{$S_{+}=124 \pm 11$} & \multicolumn{3}{c||}{$\Delta S=147 \pm 13$} & \multicolumn{1}{c}{} \\
\hline
\end{tabular}

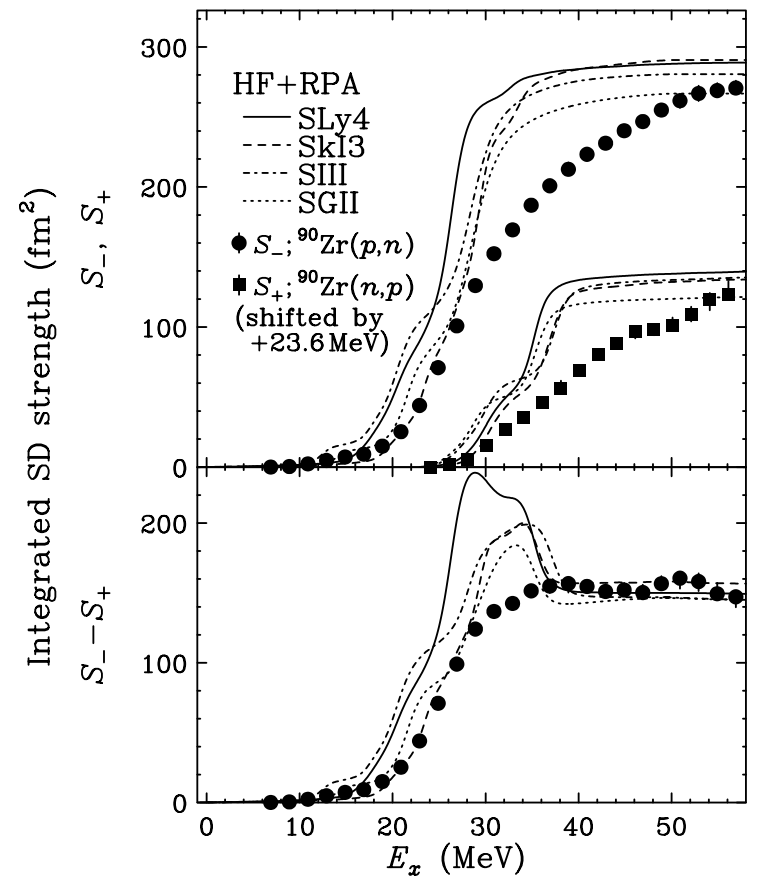

Fig. 9. Integrated charge exchange SD strength 34 excited by the operators $\hat{S}_{-}$and $\hat{S}_{+}$in Eq. 31 on ${ }^{90} \mathrm{Zr}$. The calculated results are obtained by the HF+RPA model using the Skyrme interactions SIII, SGII, SLy4 and SkI3 [59]. The upper panel shows the $S_{-}$and $S_{+}$strength, while the lower panel shows the $S_{-}-S_{+}$strength. All strengths for the three multipoles $\lambda^{\pi}=0^{-}$, $1^{-}$and $2^{-}$are summed up in the results. The experimental data are taken from Ref. [56]. No quenching factor is introduced in the calculation of the integrated strength. above $E_{x}=40 \mathrm{MeV}$, while the empirical values $S_{-}$and $S_{+}$ themselves increase gradually above $E_{x}=40 \mathrm{MeV}$. This is the crucial feature for extracting the model-independent sum rule $\Delta S=S_{-}-S_{+}$from the experimental data. The empirical values $S_{-}, S_{+}$and $\Delta S$ obtained from these analyses are shown in Table 1. The indicated uncertainties of $S_{-}, S_{+}$and $\Delta S$ contain not only the statistical error of the data, but also errors due to the various input of the DWIA calculations used in the MDA, such as the optical model parameters and the single-particle potentials [55]. There is an additional uncertainty in the estimation of the SD unit cross section, namely, the overall normalization factor [56], which should be studied further experimentally.

Table 2. Proton, neutron and charge radii of ${ }^{90} \mathrm{Zr}$. The charge radius is obtained by folding the proton density with the proton finite size. The sum rule values $\Delta S=S_{-}-S_{+}$of spin-dipole excitations are calculated by Eq. (33) with the HF neutron and proton mean square radii. The experimental value of the charge radius is taken from Ref. 60], while the experimental data for $r_{n}-r_{p}$ are taken from 61,56. The radii are given in units of $\mathrm{fm}$, while the SD sum rules are given in units of $\mathrm{fm}^{2}$.

\begin{tabular}{l|c|c|c|l|l}
\hline & SIII & SGII & SkI3 & SLy4 & $\exp$ \\
\hline$r_{p}$ & 4.257 & 4.198 & 4.174 & 4.225 & 4.19 (from $\left.r_{c}\right)$ \\
$r_{c}$ & 4.321 & 4.263 & 4.240 & 4.290 & $4.258 \pm 0.008$ \\
$r_{n}$ & 4.312 & 4.253 & 4.280 & 4.287 & $4.26 \pm 0.04[56$ \\
\hline$r_{n}-r_{p}$ & 0.055 & 0.055 & 0.106 & 0.064 & $0.09 \pm 0.07[61]$ \\
& & & & & $0.07 \pm 0.04[56$ \\
\hline$\Delta S$ & 147.1 & 143.1 & 158.0 & 148.1 & \\
\hline
\end{tabular}

From $\Delta S$, the neutron radius of ${ }^{90} \mathrm{Zr}$ is extracted to be $\sqrt{\left\langle r^{2}\right\rangle_{n}}=4.26 \pm 0.04 \mathrm{fm}$ from the model-independent $\mathrm{SD}$ sum rule $(33)$, where the empirical proton radius $\sqrt{\left.<r^{2}\right\rangle_{p}}=4.19 \mathrm{fm}$ is used. The proton radius is obtained from the charge radius in Table 2 by subtracting the proton finite size correction. The experimental uncertainty in the neutron skin thickness obtained by proton scattering is rather large: $\delta_{n p}=r_{n}-r_{p}=0.09 \pm 0.07 \mathrm{fm}$. This is mainly due to the difficulty to extract the neutron radius from the analysis of the proton scattering [61]. The sum rule analysis of the SD strength determines the neutron radius with $1 \%$ accuracy, which is almost the same as that expected for the parity violation electron scattering experiment. The obtained value $r_{n}-r_{p}=0.07 \pm 0.04 \mathrm{fm}$ can be used to disentangle the neutron matter EOS by 
using the strong linear correlation between the two quantities [12, 13, 14.

Very recently, Wakasa performed MDA of $(p, n)$ reaction cross sections on ${ }^{208} \mathrm{~Pb}$ observed at RCNP, Osaka University and extracted spin-dipole strength in ${ }^{208} \mathrm{Bi}[62$. He found $100 \%$ of the calculated sum rule strength for $1^{-}$ states, and about $70 \%$ of the predicted strength for $0^{-}$ and $2^{-}$states 59 . It would be of paramount importance to perform the counter experiment ${ }^{208} \mathrm{~Pb}(n, p)$ and extract empirically the model independent sum rule $S_{-}-S_{+}$from the two charge-exchange experiments, in order to obtain the neutron skin value in ${ }^{208} \mathrm{~Pb}$.

\section{Summary}

In this paper, we have focused on the main constraints on the symmetry energy that are provided by the experimental and theoretical studies of nuclear collective vibrations. We have not been fully exhaustive on this subject, in keeping with the fact that other contributions in the present volume deal with the issues we have not discussed. Thus, our discussion has concerned the isovector dipole and isovector quadupole states, as well as the isotopic dependence of the isoscalar monpole energies.

It is quite natural to think of the residual protonneutron force sustaining the isovector collective motion as being related with the symmetry energy. However, more effort is needed to make this statement more quantitative. As for the standard GDR, it has been suggested that its energy is correlated with the value of the symmetry energy at some sub-saturation density around $0.1 \mathrm{fm}^{-3}, S(0.1)$ if medium-heavy nuclei are considered. It is remarkable that the isovector GQR can be shown to lead to a consistent value of $S(0.1)$. Also, the combination of $J$ and $L$ that can be deduced is nicely consistent with other kinds of (completely independent) analysis that are presented in this volume: Eqs. (9), (13) and (14) substantiate these statements.

We have also discussed the role played by the pygmy states, or resonances. Empirically, a correlation of their fraction of EWSR with the slope parameter $L$ has been found, and reasonable values of $L \sqrt{10}$ and of the neutron skin (11) have been extracted. It is puzzling, though, that the PDR does not display in all the considered models a clear character related to the pure skin mode. This is one of the issues deserving further investigation.

All these observables do not seem capable of constraining the parameter $K_{\tau}$, associated with the second derivative of the symmetry energy. However, a completely different observable namely the dependence of the isoscalar monopole energy along an isotopic chain, can provide such a constrain. We have discussed the theoretical arguments behind that, and the measurements in the Sn and Cd isotopic chains that led to $K_{\tau}$ around $-550 \mathrm{MeV}$ (with a significant error bar still). We have also illustrated the correlations emerging from our theoretical study between $K_{\tau}$ and the other parameters associated with the symmetry energy or, more generally, with the equation of state.
A further source of information on the symmetry energy is the charge-exchange spin-dipole resonance. In fact, the sum rule obtained from the difference between the total strength in the $t_{-}$channel and the total strength in the $t_{+}$channel is proportional to the difference $N\left\langle r_{n}^{2}\right\rangle-Z\left\langle r_{p}^{2}\right\rangle$. Experiments aimed at extracting the neutron skin have been first performed in the $\mathrm{Sn}$ isotopes. More recently, in ${ }^{90} \mathrm{Zr}$, it has been possible to extract quantitatively the values of the total strengths and of the skin. It would be highly desirable to consider the case of ${ }^{208} \mathrm{~Pb}$ as well, in keeping with the fact that much effort is devoted to the study of this nucleus by using also parity-violating asymmetry measurements.

In conclusion, the study of giant resonances has been shown to provide some robust conclusions about the symmetry energy and its density dependence around nuclear matter saturation density. It is not completely evident how to improve on these first conclusions. Exploring nuclei with larger proton-neutron asymmetry (unstable nuclei) is of paramount importance as the results may either confirm the present findings or lead to some surprise. At the same time, further theoretical work is probably needed in order to assess which correlations with the EoS parameters are genuine, and which are somehow an artefact of a specific ansatz built in the energy functional.

\section{Acknowledgements}

This work has been supported in part by the U.S. National Science Foundation (Grant Nos. PHY07-58100 and PHY1068192).

\section{References}

1. M. Bender, P.-H. Heenen, P.-G. Reinhard, Rev. Mod. Phys. 75,121 (2003).

2. I. Vidaña et al., Phys. Rev. C80, 045806 (2009).

3. L. Trippa, G. Colò, E. Vigezzi, Phys. Rev. C77, 061304(R) (2008).

4. E. Lipparini, S. Stringari, Phys. Rep. 103, 1975 (1989).

5. G. Colò, N. Van Giai, H. Sagawa, Phys. Lett. B363, 5 (1995).

6. S. S. Dietrich and B. L. Berman, At. Data Nucl. Data Tables 38, 199 (1988)

7. A. Klimkiewicz et al., Phys. Rev. C76, 051603(R) (2007).

8. N. Paar, D. Vretenar, E. Khan, G. Colò, Rep. Progr. Phys. $70(2007) 691$.

9. D. Savran, T. Aumann, A. Zilges, Prog. Part. Nucl. Phys. 70, 210 (2013).

10. O. Wieland et al., Phys. Rev. Lett. 102, 092502 (2009).

11. A. Carbone, G. Colò, A. Bracco, L. Cao, P.F. Bortignon, F. Camera, and O. Wieland, Phys. Rev. C81, 041301(R) (2010).

12. B. A. Brown, Phys. Rev. Lett. 85, 5296 (2000); S. Typel and B. A. Brown, Phys. Rev. C64, 027302(R) (2001).

13. R.J. Furnstahl, Nucl. Phys. A 706, 85 (2002).

14. S. Yoshida and H. Sagawa, Phys. Rev. C69, 024318(2004).

15. Y. Suzuki, K. Ikeda, H. Sato, Prog. Theor. Phys. 83, 180 (1980). 
16. W. Nazarewicz and P.-G. Reinhard, Phys. Rev. C81, 051303(R) (2010).

17. X. Roca-Maza, G. Pozzi, M. Brenna, K. Mizuyama, and G. Colò, Phys. Rev. C85, 024601 (2012).

18. D. Vretenar, Y.F. Niu, N. Paar, and J. Meng, Phys. Rev. C85, 044317 (2012).

19. D. Vretenar, N. Paar, P. Ring, and T. Nikšić, Phys. Rev. C65, 021301(R) (2001).

20. A. Repko, P.-G. Reinhard, V.O. Nesterenko, and J. Kvasil, Phys. Rev. C87, 024305 (2013).

21. T. Inakura, T. Nakatsukasa, K. Yabana, Phys. Rev. C84, 021302 (2011).

22. T. Inakura, T. Nakatsukasa, K. Yabana, Prog. Theo. Phys. Suppl. 196, 375 (2012).

23. J. Piekarewicz, B.K. Agrawal, G. Colò, W. Nazarewicz, N. Paar, P.-G. Reinhard, X. Roca-Maza, and D. Vretenar, Phys. Rev. C85, 041302 (2012).

24. X. Roca-Maza, M. Brenna, G. Colò, M. Centelles, X. Viñas, B.K. Agrawal, N. Paar, D. Vretenar, J. Piekarewicz, Phys. Rev. C88, 024316 (2013).

25. S.S. Henshaw, M.W. Ahmed, G. Feldman, A.M. Nathan, H.R. Weller, Phys. Rev. Lett. 107, 22501 (2011).

26. X. Roca-Maza, M. Brenna, B.K. Agrawal, P.F. Bortignon, G. Colò, L. Cao, N. Paar, and D. Vretenar, Phys. Rev. C87, 034301 (2013).

27. X. Roca-Maza, G. Colò, and H. Sagawa, Phys. Rev. C86, 031306(R) (2012).

28. J. P. Blaizot, Phys. Rep. 64, 171 (1980).

29. S. Stringari, Phys. Lett. B 108, 232 (1982).

30. J. Treiner et al., Nucl. Phys. A 371, 253 (1981).

31. H. Sagawa et al., Phys. Rev. C 76, 034327 (2007).

32. Lie-Wen Chen et al., Phys. Rev. C 80, 014322 (2009).

33. D. Patel et al., Phys. Lett. B 718, 447 (2012).

34. S. Yoshida and H. Sagawa, Phys. Rev. C73, 044320 (2006).

35. S. Yoshida, private communication.

36. M. Fujiwara et al., Nucl. Instrum. Meth. Phys. Res. A 422, 484 (1999).

37. D.H. Youngblood et al., Phys. Rev. C 69, 031305 (2004).

38. B. Bonin et al., Nucl. Phys. A 430, 349 (1984).

39. M. Itoh et al., Phys. Rev. C 68, 064602 (2003).

40. T. Li et al., Phys. Rev. Lett. 99, 162503 (2007).

41. T. Li et al., Phys. Rev. C 81, 034309 (2010).

42. B. K. Agrawal, S. Shlomo, and V. Kim Au, Phys. Rev. C 68, 031304 (2003).

43. G. Colò et al., Phys. Rev. C 70, 024307 (2004).

44. B. G. Todd-Rutel and J. Piekarewicz, Phys. Rev. Lett. 95, 122501 (2005).

45. S. Shlomo, V.M. Kolomietz, and G. Colò, Eur. Phys. J. A 30, 23 (2006).

46. G. Colò, Phys. Part. Nuclei 39, 286 (2008).

47. J. Li, G. Colò, and J. Meng, Phys. Rev. C 78, 064304 (2008).

48. P. Veselý, J. Toivanen, B. G. Carlsson, J. Dobaczewski, N. Michel, and A. Pastore, Phys. Rev. C 86, 024303 (2012).

49. L. Cao, H. Sagawa, and G. Colò, Phys. Rev. C 86, 054313 (2012)

50. S. K. Patra et al., Phys. Rev. C 65, 044304 (2002).

51. J. Piekarewicz and M. Centelles, Phys. Rev. C 79, 054311 (2009).

52. J.M. Pearson, N. Chamel, and S. Goriely, Phys. Rev. C 82, 037301 (2010).

53. C. Gaarde et al., Nucl. Phys. A369, 258 (1981).
54. T. Wakasa, H. Sakai, H. Okamura, H. Otsu, S. Fujita, S. Ishida, N. Sakamoto, T. Uesaka, and Y. Satou, M. B. Greenfield and K. Hatanaka, Phys. Rev. C55, 2909(1997).

55. K. Yako et al., Phys. Lett. B615, 193(2005).

56. K. Yako, H. Sagawa and H. Sakai, Phys. Rev. C74 ,051303(R) (2006).

57. M. Ichimura, H. Sakai and T. Wakasa, Prog. Part. and Nucl. Phys. 56, 446 (2006).

58. A. Krasznahorkay et al., Phys. Rev. Lett. 82, 3216(1999). 59. H. Sagawa, S. Yoshida, Xian-Rong Zhou, K. Yako and H. Sakai, Phys. Rev. C76, 024301(2007).

60. H. de Vries, C. W. de Jager and C. de Vries. Atomic Data and Nuclear Data Tables 36, 495(1987).

61. L. Ray, G. W. Hoffman, G, S. Blanpied, W. R. Coker and R. P. Liljestrand, Phys. Rev. C18, 1756 (1978).

L. Ray, G. W. Hoffman and W. R. Coker, Phys. Report 212, 223 (1992).

62. T. Wakawa, a talk in RCNP workshop on "Importance of Tensor Interactions in Nuclei and Hadron structure" (July 11-12, 2013, RCNP, Osaka, Japan) T. Wakasa et al., Phys. Rev. C85, 064606 (2012). 